\title{
The application of Monte Carlo simulation in European call option pricing
}

\author{
Ze-Wei Zhang ${ }^{1, \text { a }}$, Kun Liu, Zi-Xuan Cao, \\ Zhuo Yang, Zi-Ting Luo, Zhi-Gang Zhang \\ ${ }^{1}$ University of Science \& Technology Beijing, China \\ a18810570225@163.com
}

Keywords: Monte Carlo simulation; option pricing; Wiener process.

\begin{abstract}
Monte Carlo simulation is an effective method to solve the problem of option pricing. This article introduces the definition of option, the classification of option according to different standards and methods to evaluate the option. Then it focuses on the problem of how to use the method of Monte Carlo simulation to solve the option valuation of the European call option. Next it analyzes the algorithm of Wiener process, the algorithm of stock price model, and the algorithm of European call option. Finally it changes some parameters to observe its effect on option valuation. This series of steps show how to use the Monte Carlo simulation method to value the option in detail. After reading this article, even if a beginner can have a preliminary understanding of the options, and write some relevant programs based on this article.
\end{abstract}

\section{Summary}

\subsection{The definition of the option}

An option is a contract that gives the buyer the right to buy or sell a certain amount of the underlying asset at a price that is agreed by both parties within the due date. The main element of an option contract are the underlying asset, the maturity date, and the strike price. Option pricing is the primary problem of options trading, Black-Scholes option pricing formula is very the famous.

\subsection{The description of the option}

According to the buyer's rights, options can be divided into the call option and put option. Any contract that permits the buyer to buy the underlying asset option is a call option; and any contract that permits the buyer to sell the underlying asset rights is a put option. Clearly, the buyer of the call option expects the price of the underlying asset to rise, while the buyer of the put option expects the price of the underlying asset to decline.

In accordance with the buyer to implement the option of the time limit, the option can be divided into European options and American options. European options can only be exercised at the option expiry date. While the American option allows the buyer to exercise the option at any time before the option expires.

\subsection{The option pricing methods}

At present, the main results of the study on option pricing methods are traditional option pricing method, Black-Scholes option pricing method, binary tree option pricing method, finite difference method, Monte Carlo simulation method, deterministic arbitrage method and interval pricing method. This paper focuses on how to use the Monte Carlo simulation method to solve the option pricing problem.

\section{The Monte Carlo Simulation of European Call Option Pricing}

Here is our idea, first we use the software to simulate Wiener random process, and then we use a lot of random processes and the known stock pricing formula to simulate a series of stock price curve. Finally, by using a large number of stock price curves to compute the average income, the expected 
pricing of European call option can be obtained by using simulation method. Next, we will elaborate on these three parts of the simulation method.

\subsection{The simulation method of Wiener process}

First, we introduce the definition of Wiener process. Let $\{\mathrm{W}(\mathrm{t}), \mathrm{t} \geq 0\}$ be a stochastic process, if it satisfy the requirements blow

(1) Orbital continuity $\mathrm{W}(0)=0, \mathrm{~W}(\mathrm{t})$ is a continuous function of $\mathrm{t}$,

(2) The increment obeys the normal distribution For fixed $t, \mathrm{~W}(\mathrm{t}) \sim \mathrm{N}\left(0, \sigma^{2} t\right)$, and for $\mathrm{t}>\mathrm{s}$, we have $\mathrm{W}(\mathrm{t})-\mathrm{W}(\mathrm{s}) \sim \mathrm{N}\left(0, \sigma^{2}(t-s)\right)$

(3) Increments are independent $\quad$ For arbitrary $0<t_{1}<t_{2}<\cdots<t_{n}, \mathrm{~W}\left(t_{n}\right)-W\left(t_{n-1}\right), W\left(t_{n-1}\right)-W\left(t_{n-2}\right), \ldots, W\left(t_{2}\right)-W\left(t_{1}\right), \mathrm{W}\left(t_{1}\right)$ are independent.

Then, $\{\mathrm{W}(\mathrm{t}), \mathrm{t}>0\}$ is a Brownian motion. When $\sigma=1$, it is called standard Brownian motion.

As a result, we can get the method of Wiener process's simulation by using MATLAB, specific steps are as follows:

We give the number of $\mathrm{N}$, the total time $\mathrm{T}$, and the number of segments $\mathrm{K}$ for each Wiener process. Then we can use the number of tracks to simulate the number of Wiener process, and according to the number of segments we can find the time interval $\mathrm{dt}=\mathrm{T} / \mathrm{K}$.

(i) we generate a Random matrix which has $\mathrm{N}$ rows and k columns of normal distribution $\mathrm{dW}$, the variance of normal distribution is $\mathrm{dT}$, and its expectation is 0 ,

(ii) we Initialize a matrix called $\mathrm{W}$ and record the Wiener process. And we value the first column in the $\mathrm{dW}$ to the first column in the $\mathrm{W}$,

(iii) we cycle the first and second steps. Starting from $\mathrm{i}=2$, we Add the (i-1)th columns in the W to the ith columns in the dW.

Thus, $\mathrm{W}$ is able to represent the $\mathrm{N}$ Wiener process of the matrix, in which each line represents a Wiener process.

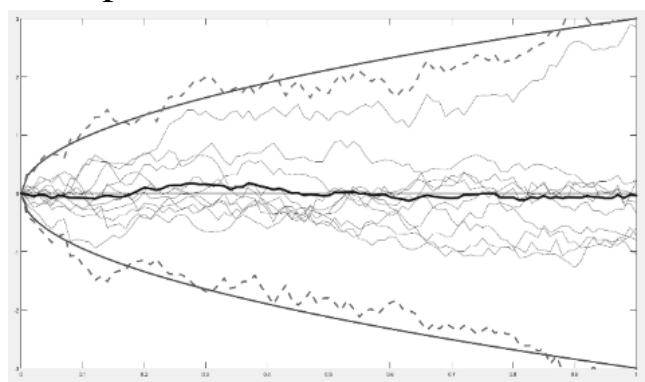

Fig. 1.1 10 Wiener stochastic processes

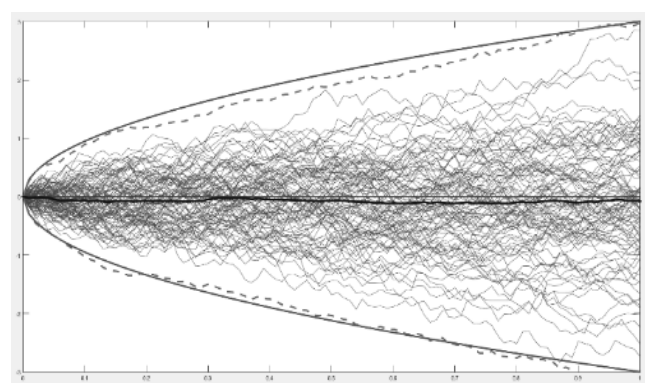

Fig. 1.2100 Wiener stochastic processes

To demonstrate the characteristics of the Wiener process better, we make 8 more curves. They are, theoretical curve of $3 \sigma$ (the outer rounded curve), Simulated Broken line of $3 \sigma$ (the dotted line), Mean curve(the thicker polyline located at the center) and theoretical expectation curve(the center line). Figure 1.1 shows the simulation image of the 10 Wiener processes. From the image we can konw that in this simulation, the mean value of 10 Wiener stochastic processes is always near the expected value, and $3 \sigma$ interval obtained by the simulation is similar with the interval obtained by theory and all the simulated curves are present in the $3 \sigma$ curve. Now we increase the number of Wiener process to 100 , as is shown in Fig. 1.2. The mean curve almost covers the curve of theoretical expectation we draw. And the simulated $3 \sigma$ interval is always around the theoretical interval. What's more, most of the curves still exist in the $3 \sigma$ interval. Obviously, the more the number of simulations, the more obvious of the regularity, and what's more, the closer to our theory.

\subsection{Stock price model}

Next, we give a simulation of the stock price:

(i) $\Delta_{t}=\frac{1}{t}$ is the time interval and get the value of it when $\mathrm{t}=\mathrm{n} \Delta_{t}+t_{0}$ which is the current time.

(ii) Get a wiener process, which is $W_{t}$, 
(iii) Through the stock pricing formula $S_{t}=S_{0} e^{\left(\mu-\frac{\sigma^{2}}{2}\right) t} E e^{\sigma W(t)}=S_{0} e^{\left(\mu-\frac{\sigma^{2}}{2}\right) t} e^{\frac{\sigma^{2} t}{2}}=S_{0} e^{\mu t}$, we can get the expectations of stock pricing theory,

(iv) Get the actual simulation of average stock price,

(v) Through the formula $E S_{t}=S_{0} e^{\left(\mu-\frac{\sigma^{2}}{2}\right) t} E e^{\sigma W(t)}=S_{0} e^{\left(\mu-\frac{\sigma^{2}}{2}\right) t} e^{\frac{\sigma^{2} t}{2}}=S_{0} e^{\mu t}$, we can get the theoretical expectations of stock price.

Finally, draw the image and plot the $3 \sigma$ curve, which is shown in Figure 2, where the abscissa represents time and the ordinate represents the stock price.

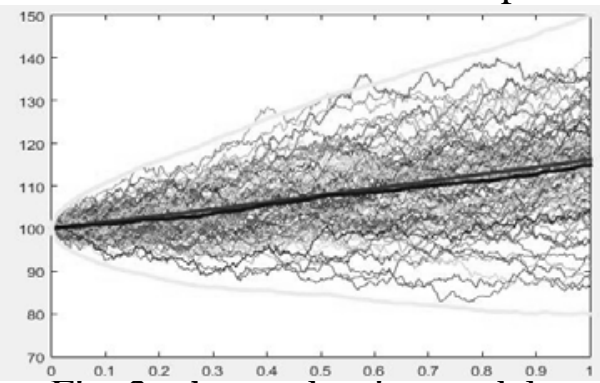

Fig. 2. the stock price model

As can be seen from Figure 2, the mean curve of the stock price is almost coincident with the theoretical expectation curve of the yield under the bank risk-free interest rate and almost all curves are in the interval of $3 \sigma$.

\subsection{Simulation of European Call Option}

European call options specific algorithm is as follows:

(i) Stock price volatility is $\sigma$; the initial price of the stock is $S_{0}$;the stock's strike price is $\mathrm{X}$; Risk free rate is $r$ which is equal to $u$,

(ii) Get the value of the stock price at time T called ST,

(iii) ST is compared with the execution price $\mathrm{X}$ and we compute the equation (ST-X)+ $=$ maximum $(\mathrm{ST}-\mathrm{X}, 0)$,

(iv) Determine the value of a simulation process, $\mathrm{Vi}=\exp (-\mathrm{rT})^{*}(\mathrm{ST}-\mathrm{X})+$,

(v) Find the cumulative average of the pricing,

(vi) Simulate the stock price changes,

(vii) Calculate the actual European call option pricing.

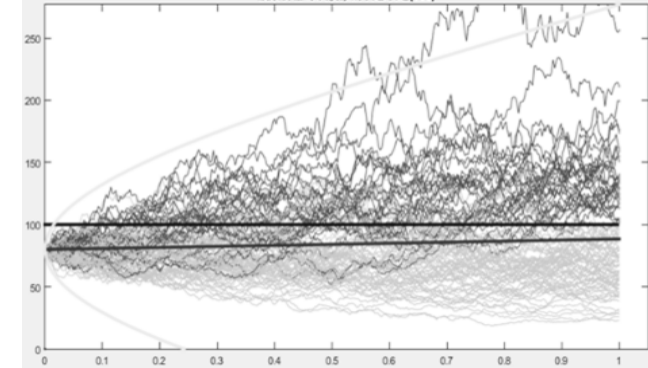

Fig. 3. stock price simulation

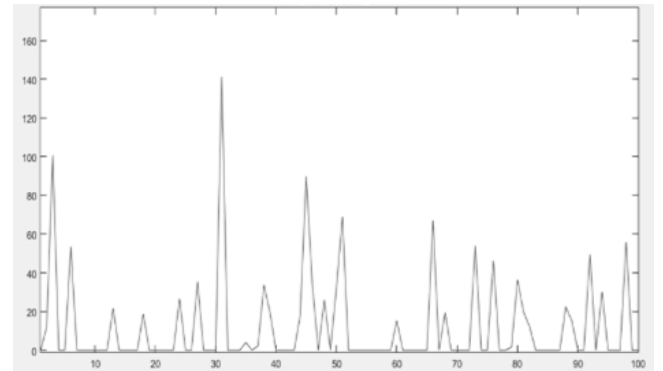

Fig. 4. option pricing simulation

Figure 3 is the 100 stock pricing simulation, Where the abscissa represents time and the ordinate represents the stock price. The parameter value-sigma is equal to 0.5 , The parameter value- $S_{0}$ is equal to 80 , The parameter value- $X$ is equal to 100 , The parameter value- $r$ is equal to 0.1 .

From top to bottom, the two curves represent the strike price and the expected return at the riskfree rate. If the end of the broken line is above the strike price curve, it indicates that the final stock price is greater than or equal to the strike price and stock returns are greater than zero. If the end of the broken line is under the strike price curve, it indicates that the final stock price is less than or equal to the strike price and stock returns are equal to zero.

Figure 4 shows the curve of the option price for each simulation based on the final stock price, in which the abscissa represents the simulation of the number of times, and the ordinate represents the option price. 


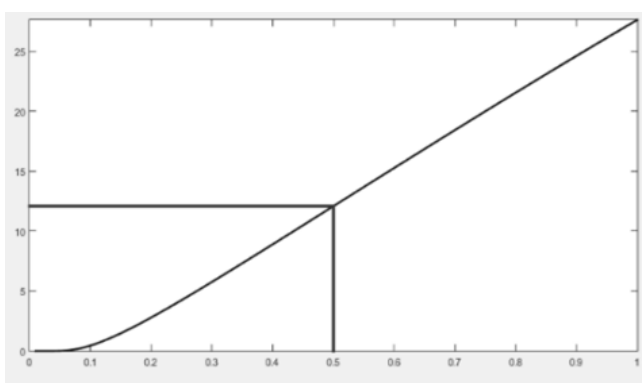

Fig. 5. option theory pricing curve

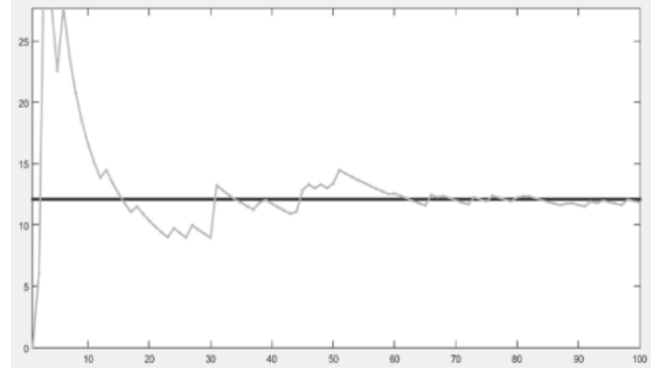

Fig. 6. simulated income mean and theoretical pricing of European call option

Figure 5 shows the theoretical expectation curve of European call option pricing when $r=0.1$ by $\mathrm{BS}$ formula. Its abscissa is $\sigma$, and the ordinate is the theoretical price of the call option. The formula is $\mathrm{V}=S_{0} \Phi_{0}\left(d_{1}\right)-X e^{-r T} \Phi_{0}\left(d_{2}\right)$, in which $d_{1}$ is equal to $\frac{\ln \left(\frac{S_{0}}{X}\right)+\left(r+\frac{\sigma^{2}}{2}\right) T}{\sigma \sqrt{T}}, d_{2}$ is equal to $d_{1}-\sigma \sqrt{T}$. Here we take $\sigma=0.5$, and obtain the expectation of pricing theory when the rate of change is 0.5 .

Figure 6 shows the curve of the theoretical option price and the cumulative average of analog pricing. As the number of simulations increases, the simulation pricing approaches theoretical pricing. The abscissa represents the number of simulations, and the ordinate represents the average option pricing.

\section{The discussion on the Pricing Formula of Call Option}

In the following, we begin to discuss the parameters of the above option pricing algorithm to verify their impact on option pricing.

\subsection{The Impact of Stock Price Fluctuation on Option Pricing}

We discuss the impact of the value of the stock price change rate $\sigma$ on the option price at first. When sigma $=0.5$, the image is shown in Fig. 3 to Fig.6. Now, we increase sigma to 0.8, and get the stock price simulation image and theoretical option pricing image.

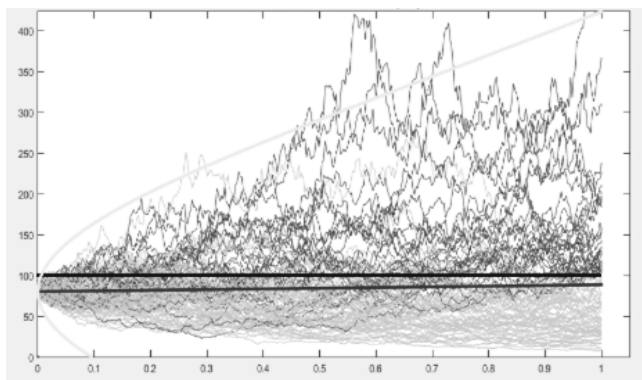

Fig. 7. stock price simulation

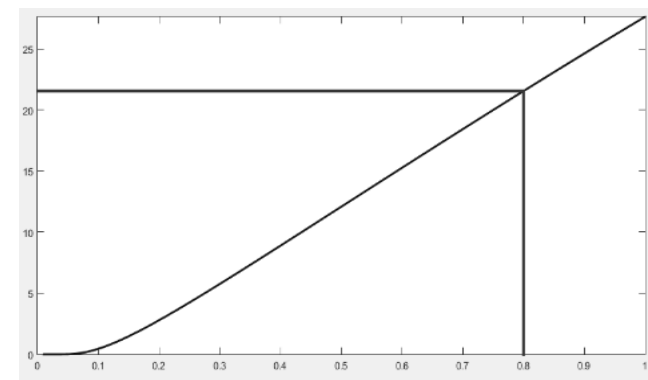

Fig. 8. option theory pricing curve

It can be seen that as the value of sigma increases, the final price of the stock is higher on the whole. And both the buyer's expected earning and the theoretical pricing of options will increase.

\subsection{The Impact of Stock Initial and Exercise Prices on Option Pricing}

The initial price of the stock price itself is also an important factor affecting the price of options. The option pricing figure for the stock is shown in Figure 3 through Figure 6, in which the initial price is 80 and the execution price is 100 .

We raise the stock's strike price to 120 at first. The stock price simulation figure and the theoretical option pricing figure under this condition are shown in Figure 9 and Figure 10.

As it can be seen, when we raise the strike price of the stock, the price of the option is reduced, which means that the buyer's expectation of earnings is also reduced.

Now we will re-adjust the execution price to 100 , change the stock's initial price to 90 , the curve is shown in Figure 11 and Figure 12. 
It can be seen that when the initial price increases, the buyer's expectation of gain is increased.

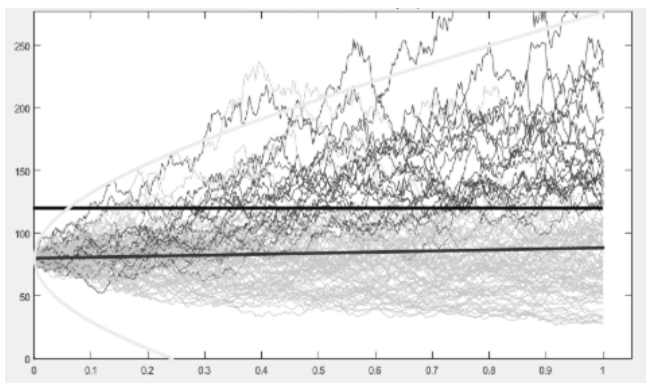

Fig. 9. stock price simulation

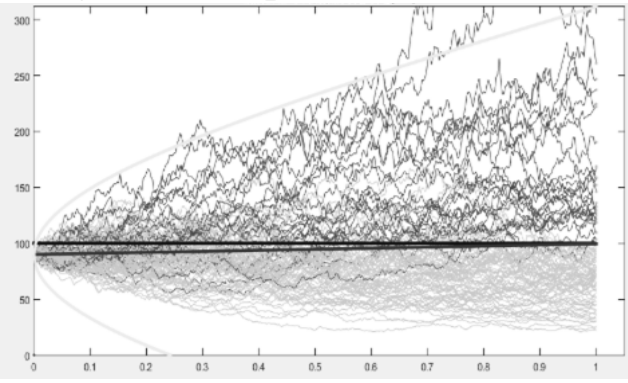

Fig.11. stock price simulation

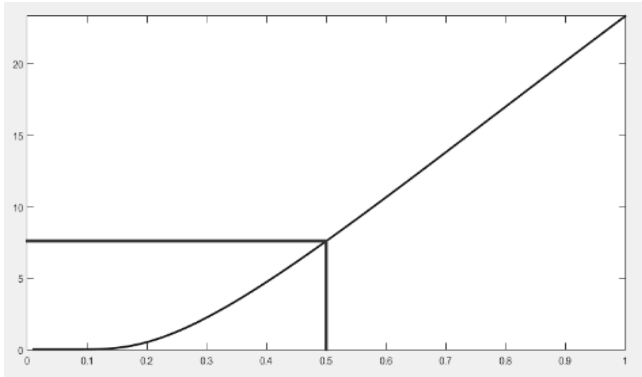

Fig. 10. option theory pricing curve

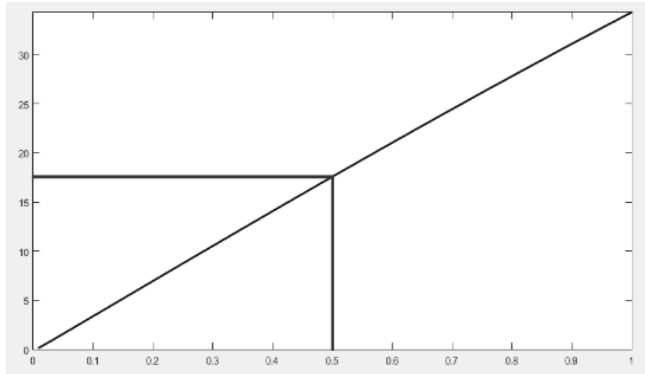

Fig. 12. option theory pricing curve

\subsection{The Impact of Risk - free Interest Rate on Option Pricing}

Interest rates are compensation for opportunity costs and risks, where compensation for opportunity costs is called the risk-free rate. The option pricing image with risk-free interest rate of 0.1 is shown in Fig. 3 to Fig.6.Then, the risk-free interest rate is increased to 0.2, the stock pricing simulation image and the call option pricing theory expected image are shown in Fig.13 and Fig.14.

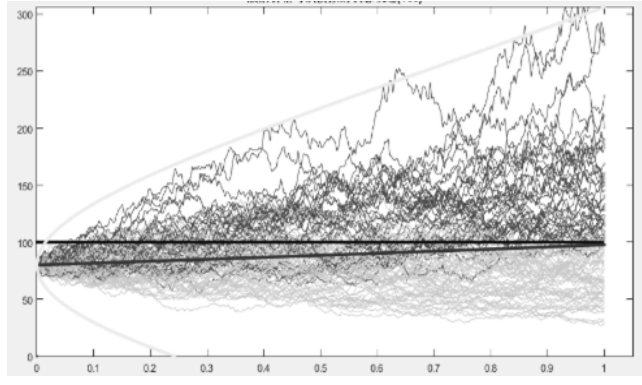

Fig.13. stock price simulation

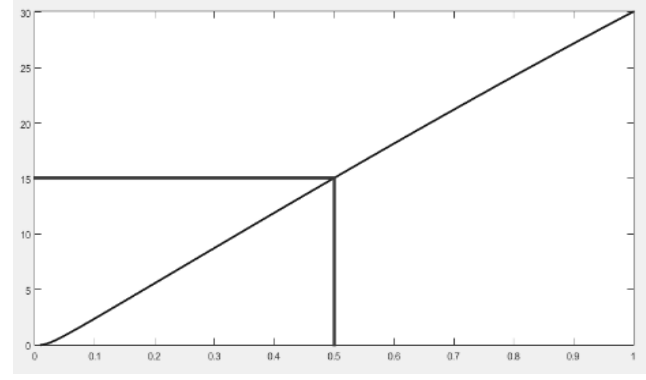

Fig. 14. option theory pricing curve

Through the image, we can see that, when the bank increases the risk-free interest rate, the stock value is generally higher, and the expectation of the gain is correspondingly increased, and the theoretical expectation of option pricing also increases. It is not difficult to guess that when the bank reduces the risk-free rate, the value of the stock will generally be lower and the option value will be lower.

\section{References}

1. Mou Kuangning, Comparison of Monte Carlo Simulation and Quasi Monte Carlo Simulation in Option Pricing (Science Technology and Engineering, 2002), pp. 1925-1933.

2. Liu Hailong and Wu Chongfeng, Survey of Option Pricing Methods (Journal of Management Sciences in China, 2005), pp. 67-73.

3. Chen Weiping and Zhou Yi, Research on the Application of Stock Valuation Model (Finance, 2013) , pp. 98-99.

4. Chen Jia and Wu Runheng, European Option Pricing Theory in Finance Mathematics (Journal of North China University of Technology, 2007) , pp. 75-78.

5. Huai Tao, The Research of Option Pricing Strategy and Pricing Method (Times Finance, 2013), pp. 278-280. 